\title{
Genome-wide identification of $O T P$ gene as a novel methylation marker of breast cancer
}

\author{
MYUNG SOON KIM ${ }^{1,4}$, JINSUN LEE ${ }^{2}$, TAEJEONG OH ${ }^{1}$, YOUNGHO MOON ${ }^{1}$, EILSUNG CHANG ${ }^{2}$, \\ KWANG SUN SEO ${ }^{3}$, BENJAMIN DOUGLAS HOEHN ${ }^{1}$, SUNGWHAN AN ${ }^{1}$ and JEUNG-HOON LEE ${ }^{4}$ \\ ${ }^{1}$ Genomictree Inc., Daejeon 301-721; Departments of ${ }^{2}$ Surgery and ${ }^{3}$ Pathology, Chungnam National \\ University College of Medicine, Daejeon 301-721; ${ }^{4}$ Department of Dermatology, \\ School of Medicine, Chungnam National University, Daejeon 301-721, Republic of Korea
}

Received November 30, 2011; Accepted January 12, 2012

DOI: $10.3892 / o r .2012 .1691$

\begin{abstract}
Aberrant DNA methylation occurs early and frequently in tumorigenesis. Identification of DNA methylation biomarkers is a field that provides potential for improving the clinical process of breast cancer diagnosis. We utilized a genome-wide technique, methylated DNA isolation assay (MeDIA), in combination with high-resolution $\mathrm{CpG}$ microarray analysis to identify hypermethylated genes in breast cancer. Among differentially methylated genes between tumor and adjacent normal tissues, 3 candidate genes (LHX2, WT1 and $O T P$ ) were finally selected through a step-wise filtering process and examined for methylation status in normal tissues, primary tumor, and paired adjacent normal-appearing tissues from 39 breast cancer patients. Based on the calculated cut-off values, all genes showed significantly higher frequencies of aberrant hypermethylation in primary tumors (43.6\% for $L H X 2$, $89.7 \%$ for $W T 1$ and $100 \%$ for $O T P, \mathrm{P}<0.05$ ) while frequencies were intermediate in paired adjacent normal tissues and absent in normal tissues. On further analysis, the methylation level in primary tumors was not significantly correlated with clinicopathological features. Interestingly, DNA methylation of a novel gene $O T P$ was detected in adjacent normal tissues even $6 \mathrm{~cm}$ away from primary tumors, suggesting that $O T P$ methylation may qualify as a biomarker for the early detection of breast cancer. In conclusion, we successfully identified a novel gene $O T P$ frequently methylated in breast cancer by genomewide screening. Our results suggest that the OTP gene may
\end{abstract}

Correspondence to: Dr Sungwhan An, Genomictree Inc., 829 Tamnip-dong Yuseong, Daejeon 305-501, Republic of Korea E-mail: genomictree1@korea.com

Professor Jeung-Hoon Lee, Department of Dermatology, School of Medicine, Chungnam National University, 33 Munhwa-ro, Jung-gu, Daejeon 301-721, Republic of Korea

E-mail: jhoon@cnu.ac.kr

Key words: breast cancer, OTP, methylation biomarker, early detection play a crucial role in breast carcinogenesis, although further clinical validation will be needed to evaluate the potential application of $O T P$ in the early detection of breast cancer.

\section{Introduction}

Breast cancer is the most frequently diagnosed cancer in women with 1.15 million cases reported worldwide every year $(1,2)$. Higher quality mammography and magnetic resonance imaging have increased the detection of breast cancer leading to a overall decline in mortality. These screening procedures, however, may also lead to over diagnosis and result in unnecessary treatments and procedures (http://www.breastcancer. org/). Therefore, development of new screening tools with high accuracy would be of great value.

It is now widely recognized that aberrant epigenetic modifications play a crucial role in altering gene expression and inducing tumor formation $(3,4)$. In recent years much attention has been paid to epigenetic gene silencing. This loss of gene transcription is due to hypermethylation of $\mathrm{CpG}$ islands in the promoter region and is known to frequently occur in the early stages of cancer development. These findings have suggested that such aberrant events could be utilized as molecular diagnostic biomarkers for early detection or risk identification for cancers (5).

There have been several studies profiling gene methylation at a genomic level or using panels of known genes (6-11). These studies have attempted to define methylation sites in genes and determine their frequency of methylation in clinical samples, which may lead to their use in molecular diagnostic testing for early detection of breast cancer. However, these established cancer-associated genes do not yet have a sufficient usability to be considered efficient clinical diagnostic markers.

In the present study, we performed a $\mathrm{CpG}$ microarray analysis in conjunction with a proprietary method for enrichment of methylated DNA we developed, using optimally truncated methyl DNA binding domain (MBD2bt), named methylated DNA isolation assay (MeDIA) (12). We used this system to characterize frequently methylated genes in early stage primary tumors and matched normal appearing tissues from breast cancer patients. Through step-wise filtering and subsequent verification of methylation-positivity in clinical 
samples using a pyrosequencing-methylation assay, we identified new candidate genes which may provide additive value to other potential methylation biomarkers for the early detection of breast cancer.

\section{Materials and methods}

Reagents. All chemical reagents used were purchased from Sigma-Aldrich (MA, USA) unless otherwise noted. Oligonucleotides were synthesized by Bioneer Co. (Daejeon, Republic of Korea).

Cells and clinical specimens. The human breast cancer cell lines, MCF-7 (HTB-22) and MDA-MB-231 (HTB-26), were obtained from the American Type Culture Collection and cultured in RPMI-1640 supplemented with $10 \%$ fetal bovine serum.

Fresh-frozen tissue specimens were obtained from the School of Medicine, ChungNam National University, Daejeon, Republic of Korea. All specimens and pertinent patient information were treated in accordance with the Institutional Review Board of the School of Medicine, ChungNam National University, Daejeon, Republic of Korea. Each tumor specimen was histologically verified by a board-certified pathologist and archived for further DNA study. Genomic DNA of normal tissues without any history of malignancy was purchased from BioChain, Inc. (CA, USA). Patient characteristics used in study are described in Table I.

Genome-wide methylation microarray analysis. CpG microarray analysis in conjunction with the enrichment of methylated DNA by methylated DNA isolation assay (MeDIA) using optimally truncated methyl DNA binding domain (MBD2bt) was performed as previously described with slight modification (12). The genomic DNA was isolated from samples taken from breast tumor blocks and their adjacent normal tissues of 3 patients (stage 1) and the equal amount of DNA was mixed. Briefly, $0.5 \mu \mathrm{g}$ of sonic fragmented genomic DNA from primary tumor and their match-paired normal-appearing adjacent tissues was incubated with $2 \mu \mathrm{g}$ of recombinant MBD2bt protein for $4 \mathrm{~h}$ at $4^{\circ} \mathrm{C}$ with rocking platform, respectively. The enriched methylated DNA was amplified using whole genome amplification kit (GenomePlex ${ }^{\circledR}$, Sigma, USA) as recommended by the manufacturer. The common reference DNA (CRD) was prepared by amplifying genomic DNA from normal placenta control without enrichment as an internal control. Enriched methylated DNA and CRD were labeled with $\mathrm{Cy} 5$ and $\mathrm{Cy} 3$, respectively. The labeled DNA samples were purified by PCR purification kit (Qiagen, USA) and then co-hybridized to human $\mathrm{CpG}$ island microarrays containing 237,000 oligonucleotide probes covering 27,800 CpG islands (Agilent, Santa Clara Technologies, Inc., CA, USA) according to the manufacturer's instructions.

Microarray data analysis. The hybridized images were analyzed by Agilent DNA microarray Scanner (Agilent Technologies, Inc.) and the data quantification was performed using Feature Extraction software version 9.3.2.1 (Agilent Technologies, Inc.). Preprocessing of raw data and normalization steps were performed using GeneSpring 7.3.1 (Agilent
Table I. Clinicopathological features of the 39 breast cancer patients.

\begin{tabular}{lr}
\hline Features & Patients \\
\hline Age & \\
$\leq 50$ & 21 \\
$>50$ & 18 \\
Stage & \\
I & 17 \\
II & 19 \\
III & 3
\end{tabular}

Tumor size

T1 17

T2 21

T3 1

Histological grade

I

II

III

LN metastasis

pNO 25

$\mathrm{pN} 1+12$

$\mathrm{pN} 2$

1

$\mathrm{pN} 3$

1

ER

Negative $\quad 10$

Positive 29

PR

Negative $\quad 12$

Positive $\quad 27$

HR status

Positive $\left(\mathrm{ER}^{+}\right.$and/or $\left.\mathrm{PR}^{+}\right) \quad 8$

Negative $\left(\mathrm{ER}^{-}\right.$and $\left.\mathrm{PR}^{-}\right)$

HER-2/neu status

Positive 6

Negative 33

Histological type

Ductal

32

Lobulal

1

Mixed ductal and lobular $\quad 1$

Other

5

ER, estrogen receptor; PR, progesterone receptor; HR, hormone receptor.

Technologies, Inc.). The background-corrected intensity data were normalized using the intensity dependent LOWESS method to remove the dye bias within each array as recommended by the manufacturer (Agilent Technologies, Inc.). The relative methylation level of each probe between tumor and their adjacent normal tissues was calculated by comparison of ratio in each of enriched DNA/CRD. The multiple-probe 
Table II. Primers for bisulfite PCR and pyrosequencing.

\begin{tabular}{|c|c|c|c|c|}
\hline Genes & Primer $\left(5^{\prime}-3^{\prime}\right)$ & Assayed CpG sites ${ }^{\mathrm{a}}$ & $\begin{array}{l}\text { Annealing } \\
\operatorname{Tm}\left({ }^{\circ} \mathrm{C}\right)\end{array}$ & $\begin{array}{l}\text { Amplicon size } \\
\text { (bp) }\end{array}$ \\
\hline OTP & $\begin{array}{l}\text { Forward: TATTYGGAAATTGGTTTTTA } \\
\text { Reverse: Biotin-TCRATTTTAAAAAACTTCTT } \\
\text { Sequencing (F): TTATYGTTATTTTTTAATGG }\end{array}$ & $\begin{array}{l}+2064,+2073,+2079 \\
+2091,+2099\end{array}$ & 52 & 98 \\
\hline$L H X 2$ & $\begin{array}{l}\text { Forward: TYGTTTAGAAATGGAAATGGGTT } \\
\text { Reverse: Biotin-CCACAACTCCCTCTATAACCTTAACT } \\
\text { Sequencing (F): TTGGGGTGGGGGGAA }\end{array}$ & $\begin{array}{l}+2212,+2214,+2217 \\
+2239\end{array}$ & 58 & 106 \\
\hline$W T 1$ & $\begin{array}{l}\text { Forward: YGGTTTGATTYGGTAATTTAGTA } \\
\text { Reverse: Biotin-AAAAACAAAACCCTTCTC } \\
\text { Sequencing (F):TTTTTTTTGGAGTAAAATTAT }\end{array}$ & $+1991,+2006,+2024$ & 54 & 175 \\
\hline
\end{tabular}

Y, C or T, R, A or G. ${ }^{a}$ Distance (nt) from transcription start site (+1).

enriched genes were selected as methylation target genes when their probes showed positive call $\geq 2$. 0 -fold in methylation of tumor compared with paired adjacent normal tissues in at least two adjacent probes allowing a one-probe gap within the $\mathrm{CpG}$ islands.

The raw DNA microarray data were submitted for public access to the Gene Expression Omnibus (http://www.ncbi.nlm. nih.gov/geo/) and can be obtained using the accession number: GSE27374.

Methylation assessment by quantitative bisulfite-pyrosequencing. Bisulfite PCR and pyrosequencing primers were designed to amplify 3 to $5 \mathrm{CpG}$ dinucleotide sites in the target regions of genes using PSQ Assay Design software (Biotage, Sweden). Sequences of primers used and PCR condition are given in Table II. Genomic DNA (200 ng) was modified by sodium bisulfate using the EZ DNA Methylation kit (Zymo Research, CA, USA) according to the manufacturer's instructions. Bisulfite-modified DNA was amplified in a $50 \mu \mathrm{l}$ reaction with the primer set and Taq polymerase (Enzynomics, Daejeon, South Korea). The PCR amplification was run for 45 cycles with an optimal annealing temperature. The PCR products were visualized on $2 \%$ agarose gel electrophoresis by ethidium bromide staining.

Pyrosequencing was performed using the Pyro Gold kit and PSQ 96MA instrument (Qiagen, Hilden, Germany) as instructed by the manufacturer. The methylation index (MtI) of each gene in each sample was calculated as the average value of ${ }^{\mathrm{m}} \mathrm{C} /\left({ }^{\mathrm{m}} \mathrm{C}+C\right)$ for all examined $\mathrm{CpGs}$ in target regions. All experiments included samples without templates as negative control.

Statistical analysis. Statistical analysis was completed using the MedCalc software, version 9.3.2.0 (MedCalc, Mariakerke, Belgium). A P-value of $<0.05$ was considered as statistically significant. Wilcoxon Rank sum test was used to determine whether there were differences in methylation levels in tumors and adjacent normal tissues. The relationship between methylation level and clinicopathological features was analyzed by ANOVA test.

\section{Results}

Comprehensive methylation pattern analysis comparing primary breast tumors and matched-adjacent tissue. To comprehensively investigate $\mathrm{CpG}$ sites of genes which are aberrantly methylated early in breast cancer, we compared the DNA methylation status of pooled genomic DNA from three primary tumors with match-paired normal-appearing adjacent tissue (hereby, termed adjacent normal) from stage I breast cancer patients by MEDIA-CpG microarray analysis (12). Using this approach we observed that several hundred genes, including polycomb-target as a major group, demonstrated differential methylation between tumor and adjacent normal tissues. In order to reduce the number of genes and further verify methylation we adopted the following sequential filtering criteria: i) initial gene lists were chosen through an arbitrary selection process from probes in microarrays with signal intensities $\geq 2,000$ in tumor tissue; ii) methylation levels were compared in tumor tissues and paired adjacent normal tissue, selected genes had higher ( $\geq 2.0$-fold) levels of methylation in at least two adjacent probes allowing a one-probe gap within the $\mathrm{CpG}$ islands; and iii) genes not harboring $\mathrm{CpG}$ islands in the regulatory region were excluded (Fig. 1). From this, we obtained 6 candidate genes, AXIN2, LHX2, NFIX, OTP, PTGER4 and WTI for methylation assesment (Table III).

Examination of methylation status of 6 candidates in cell lines and normal tissue. We confirmed the methylation status of the 6 candidate genes derived by microarray analysis using a pyrosequencing assay in breast cancer cell lines MCF7 and MDA-MB-231 (Fig. 2A). In this assay, sodium bisulfite-treated genomic DNA is amplified by PCR using gene specific primer sets with methylation-independent sequences. We demonstrated that all genes appear to a have high level of methylation in both cell lines. We also examined the DNA methylation status in normal breast tissues from 6 healthy individuals. Three genes, AXIN2, NFIX, PTGER4 showed a relatively higher range of methylation level ( $\mathrm{MtI}>33 \%$ ) while the level of methylation of the remaining 3 genes, LHX2, OTP and WT1 were either lower range (MtI range from $11 \%$ to $20.6 \%$ ) 


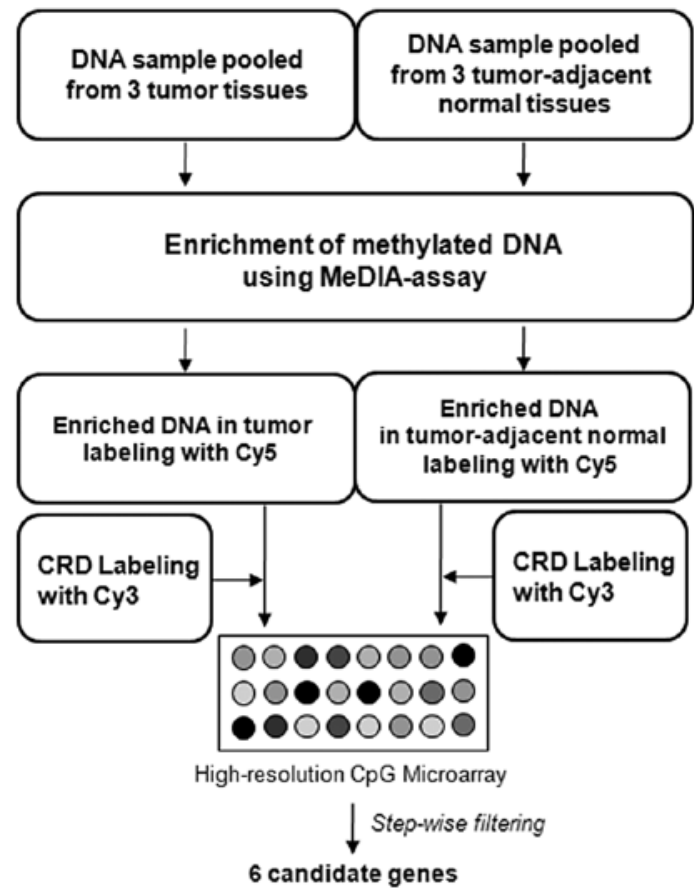

Figure 1. Schematic diagram of MeDIA-assisted high-resolution CpG microarray analysis in breast tissues. The methylated DNA was separately enriched from 3 tumors and paired adjacent normal tissues by MeDIA technique. The enriched methylated DNAs were amplified and subjected to $\mathrm{CpG}$ microarray analysis. Amplified methylated DNAs (Cy5) were individually compared with amplified-CRD (Cy3) without enrichment as an internal control. The relative methylation level of each probe across samples was calculated and 6 candidate genes were identified by step-wise filtering processes.

or unmethylated (MtI $<10 \%$ ) (Fig. 2B). We then decide not to include AXIN2, NFIX, PTGER4 genes which had Mtl $>33 \%$ in normal breast tissue, from further clinical validation studies assuming that they would not be able to meet necessary specificity standards in future testing.

Clinical validation of LHX2, OTP and WT1 genes in primary tumor and adjacent normal breast tissue. To verify whether LHX2, OTP and WT1 genes are hypermethylated in primary breast tumors, we examined their methylation status in 39 primary breast tumors and matched-adjacent normal tissue from breast cancer patients representing stages I, II, and III as well as other clinicopathological parameters (Table I). Most of the samples from cancer patients showed a higher methylation level of each gene in primary tumors when compared to matched normal tissue (61.5\% for $L H X 2,92.3 \%$ for $W T 1$ and $100 \%$ for OTP).

We then used the highest methylation levels of each gene in normal samples as a cut-off value for methylation-positive calls. All genes showed a high frequency of methylation positivity in tumors (43.6\% for LHX2, 89.7\% for WT1 and $100 \%$ for $O T P$ ). We also observed that aberrant methylation of each gene existed in tumor-adjacent normal tissue, although the levels and frequencies were intermediate between those of tumor and normal tissues (Fig. 3 and Table IV). In addition, we investigated the potential relationship between methylation levels of $L H X 2, O T P$ and $W T 1$ and clinicopathological parameters including patients' age, histological type, tumor size, histological grade, clinical stage, presence of lymph node
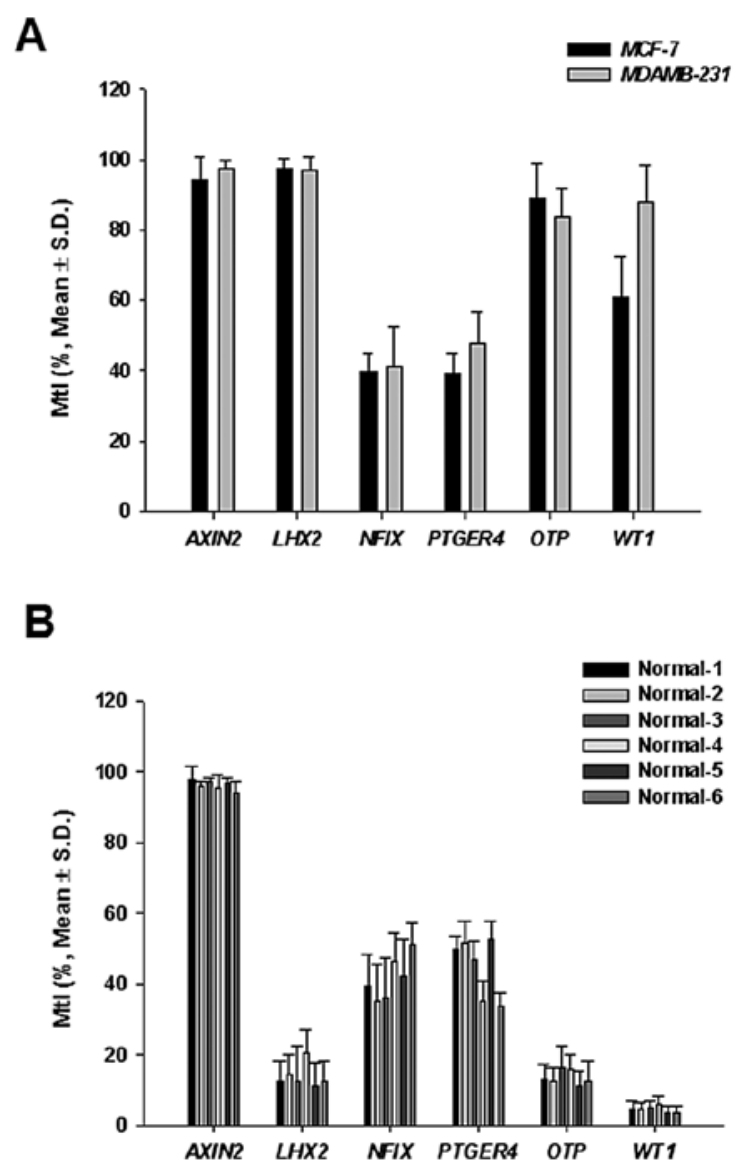

Figure 2. The quantitative pyrosequencing methylation assay of 6 candidate genes in 2 cell lines and 6 healthy normal tissues. Bisulfite-treated genomic DNA was amplified by specific primers. The MtI is presented as the mean value with standard deviation (SD) for all examined CpGs in target region. (A) Methylation status of 6 candidate genes in 2 two breast cancer cell lines, MCF-7 and MDA-MB-231. Gene names are indicated at the bottom and left axis of the methylation level, respectively. (B) The methylation levels of 6 genes in healthy normal tissues. The left axis indicates methylation level, and gene names are indicated at the bottom.

metastases, steroid receptor (ER and PR) status and HER2 amplification. There were no significant correlations between methylation levels and any of the analyzed clinicopathological parameters (Table V).

\section{Discussion}

The number of genes showing aberrant methylation in breast cancer is increasing, although only a few genes have demonstrated promise as methylation markers for early detection and risk assessment $(13,14)$. To expand these findings to novel genes in breast cancer, both gene-specific and genomic-level methylation profiling approaches have been performed (6-11).

From among those approaches, Pfeifer and colleagues focused on finding new genes which are frequently found to be methylated in early on-set breast cancer. To accomplish this they used $\mathrm{CpG}$ microarray analysis in conjunction with the methylated $\mathrm{CpG}$ island recovery assay (MIRA), which is based on the affinity of a two protein complex (MBD2/ MBD3L1) attached to methylated DNA. Their data highlighted homeobox genes such as THX1, HOXB13 and HNF1B 
Table III. Candidate genes identified by MeDIA-assisted CpG microarray analysis.

\begin{tabular}{|c|c|c|c|}
\hline Gene name & Description & Chr. location & Function \\
\hline AXIN2 & Axin 2 (conductin, axil) & $17 q 23-q 24$ & Multicellular organismal development \\
\hline$L H X 2$ & LIM homeobox 2 & $9 q 33.3$ & Regulation of transcription, DNA-dependent \\
\hline NFIX & $\begin{array}{l}\text { Nuclear factor } \mathrm{I} / \mathrm{X} \\
\text { (CCAAT-binding transcription factor) }\end{array}$ & $19 \mathrm{p} 13.3$ & $\begin{array}{l}\text { DNA replication; regulation of transcription, } \\
\text { DNA-dependent }\end{array}$ \\
\hline PTGER4 & $\begin{array}{l}\text { Prostaglandin E receptor } 4 \\
\text { (subtype EP4) }\end{array}$ & $5 \mathrm{p} 13.1$ & G-protein signaling \\
\hline OTP & Orthopedia homeobox & $5 q 13.3$ & $\begin{array}{l}\text { Multicellular organismal development; } \\
\text { regulation of transcription, DNA-dependent; } \\
\text { induction of apoptosis }\end{array}$ \\
\hline$W T 1$ & Wilms tumor $1 \mathrm{c}$ & $11 \mathrm{p} 13$ & $\begin{array}{l}\text { Negative regulation of cell proliferation; } \\
\text { epithelial cell differentiation; } \\
\text { germ cell development }\end{array}$ \\
\hline
\end{tabular}

Table IV. Methylation levels and frequencies of hypermethylation of 3 genes in 39 pairs of breast tissues.

\begin{tabular}{|c|c|c|c|c|c|}
\hline \multirow[b]{2}{*}{ Gene } & \multicolumn{2}{|c|}{ Methylation index $(\%, \text { mean } \pm S D)^{a}$} & \multicolumn{3}{|c|}{ Number and percentage of hypermethylation ${ }^{b}$} \\
\hline & Paired tumor-adjacent tissues & Tumor tissues & Normal (\%) & Paired tumor-adjacent tissues (\%) & Tumor tissues $(\%)$ \\
\hline OTP & $27.0 \pm 11.8$ & $56.2 \pm 16.3$ & $0 / 6(0.0)$ & $34 / 39(87.2)$ & $39 / 39(100)$ \\
\hline$L H X 2$ & $16.0 \pm 8.8$ & $22.7 \pm 17.2$ & $0 / 6(0.0)$ & $8 / 39(20.5)$ & $17 / 39(43.6)$ \\
\hline$W T 1$ & $6.8 \pm 3.3$ & $17.3 \pm 12.2$ & $0 / 6(0.0)$ & $23 / 39(59.0)$ & $35 / 39(89.7)$ \\
\hline
\end{tabular}

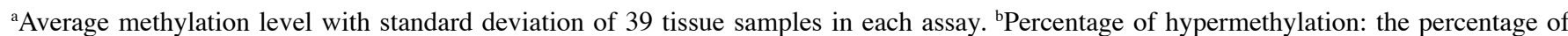
methylation-positive samples that showed higher methylation level than cut-off value. Each cut-off value was determined as the highest MtIs of each gene in 6 normal samples $(O T P, 16.6$; LHX2, 20.6 and WT1, 5.9). P-values were calculated with the use of the Wilcoxon Rank sum test $(\mathrm{P} \leq 0.05)$.

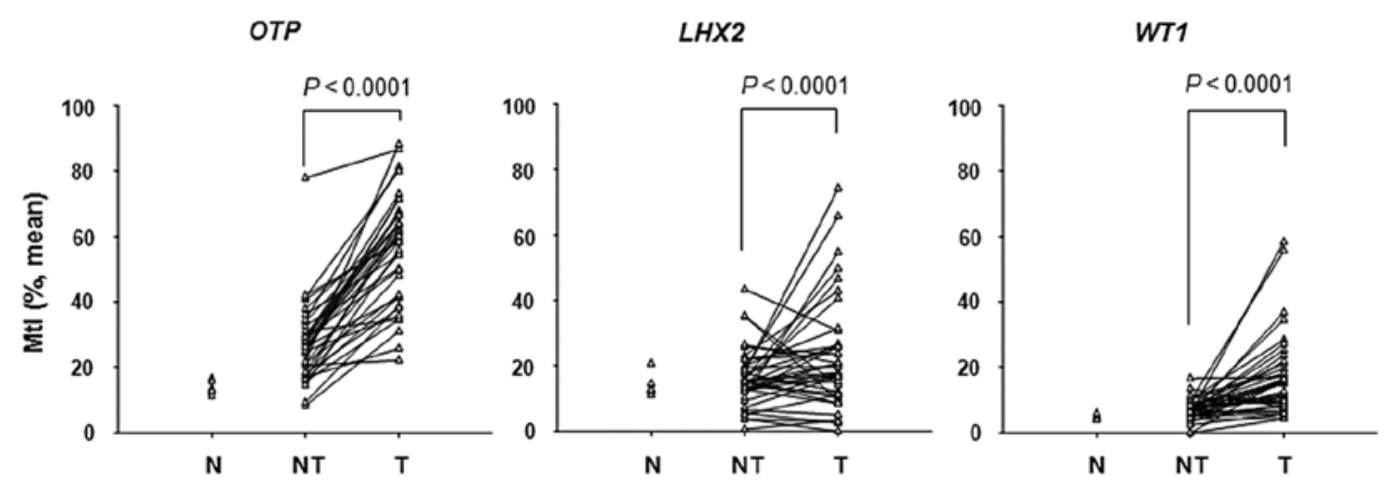

Figure 3. Methylation status of $L H X 2, O T P$ and $\mathrm{WT1}$ in healthy normal and breast tissues from breast cancer patients. Multiple line/scatter plots of MtIs for $O T P, L H X 2$ and $W T 1$ were plotted from pyrosequencing analysis of 39 clinical tumor tissue samples (T) and 39 paired tumor-adjacent normal tissue samples (NT) and 6 healthy normal tissues (N). The samples from the same patient are linked with a straight line.

as new potential methylation biomarkers and demonstrated a high frequency of methylation in significant portion of breast cancers (10).

In the present study, we employed a similar approach to screen differentially methylated $\mathrm{CpG}$ sites in primary tumor tissues and tumor-adjacent normal tissue of early stage breast cancer patients. However, we used an optimally truncated methylated DNA binding domain for the enrichment of methylated DNA (MBD2bt) instead of the MBD2/MBD3L1 complex (12). We found that hundreds of genes are aberrantly 
Table V. The relationship between clinicopathological parameters and MtIs of 3 genes in breast cancer samples.

\begin{tabular}{|c|c|c|c|c|c|c|c|}
\hline \multirow[b]{2}{*}{ Features } & \multicolumn{6}{|c|}{$\operatorname{MtIs}(\%, \text { mean } \pm \mathrm{SD})^{\mathrm{a}}$} & \multirow[b]{2}{*}{ Case } \\
\hline & OTP & P-value ${ }^{a}$ & $L H X 2$ & P-value ${ }^{a}$ & $W T 1$ & P-value ${ }^{a}$ & \\
\hline \multicolumn{8}{|l|}{ Age (mean, range) } \\
\hline$\leq 50$ & $55.9 \pm 15.8$ & 0.92 & $22.6 \pm 16.9$ & 0.95 & $14.4 \pm 10.8$ & 0.22 & 21 \\
\hline$>50$ & $56.4 \pm 17.4$ & & $22.9 \pm 18.0$ & & $20.7 \pm 13.2$ & & 18 \\
\hline \multicolumn{8}{|l|}{ Stage } \\
\hline I & $54.2 \pm 13.3$ & 0.51 & $22.2 \pm 15.1$ & 0.85 & $15.2 \pm 8.8$ & 0.35 & 17 \\
\hline II-III & $57.7 \pm 18.5$ & & $23.2 \pm 19.0$ & & $18.9 \pm 14.3$ & & 22 \\
\hline \multicolumn{8}{|l|}{ Tumor size } \\
\hline $\mathrm{T} 1$ & $56.6 \pm 13.3$ & 0.51 & $27.8 \pm 19.0$ & 0.11 & $15.2 \pm 8.7$ & & 17 \\
\hline $\mathrm{T} 2-3$ & $57.7 \pm 18.5$ & & $18.9 \pm 14.9$ & & $18.9 \pm 14.4$ & 0.35 & 22 \\
\hline \multicolumn{8}{|l|}{ Histological grade } \\
\hline I & $53.8 \pm 9.2$ & 0.26 & $28.3 \pm 21.4$ & 0.57 & $14.1 \pm 7.5$ & 0.23 & 8 \\
\hline II & $61.3 \pm 16.7$ & & $22.4 \pm 12.9$ & & $21.3 \pm 12.6$ & & 16 \\
\hline III & $52.0 \pm 18.2$ & & $20.2 \pm 19.2$ & & $14.7 \pm 13.2$ & & 15 \\
\hline \multicolumn{8}{|l|}{ LN metastasis } \\
\hline pNO & $55.8 \pm 13.8$ & 0.86 & $21.0 \pm 13.3$ & 0.41 & $17.3 \pm 12.3$ & 0.99 & 25 \\
\hline $\mathrm{pN} 1-3$ & $56.8 \pm 20.7$ & & $25.8 \pm 22.8$ & & $17.3 \pm 12.5$ & & 14 \\
\hline \multicolumn{8}{|l|}{ ER } \\
\hline Negative & $51.8 \pm 18.9$ & 0.34 & $22.5 \pm 22.7$ & 0.97 & $16.0 \pm 14.8$ & 0.70 & 10 \\
\hline Positive & $57.7 \pm 15.5$ & & $22.8 \pm 15.3$ & & $17.7 \pm 11.5$ & & 29 \\
\hline \multicolumn{8}{|l|}{ PR } \\
\hline Negative & $52.4 \pm 21.5$ & 0.34 & $25.7 \pm 22.0$ & 0.48 & $14.7 \pm 13.9$ & 0.38 & 12 \\
\hline Positive & $57.8 \pm 13.6$ & & $21.4 \pm 14.8$ & & $18.5 \pm 11.5$ & & 27 \\
\hline \multicolumn{8}{|l|}{ HR status } \\
\hline Positive $\left(\mathrm{ER}^{+}\right.$and/or $\left.\mathrm{PR}^{+}\right)$ & $52.3 \pm 20.4$ & 0.47 & $27.1 \pm 23.3$ & 0.43 & $17.1 \pm 16.6$ & 0.96 & 8 \\
\hline Negative (ER- and $\mathrm{PR}^{-}$) & $57.1 \pm 15.4$ & & $21.6 \pm 15.5$ & & $17.3 \pm 11.2$ & & 31 \\
\hline \multicolumn{8}{|l|}{ HER-2/neu status } \\
\hline Negative & $56.1 \pm 16.6$ & 0.94 & $23.2 \pm 17.6$ & 0.73 & $16.9 \pm 10.7$ & 0.67 & 33 \\
\hline Positive & $56.6 \pm 16.4$ & & $20.5 \pm 15.6$ & & $19.3 \pm 19.8$ & & 6 \\
\hline
\end{tabular}

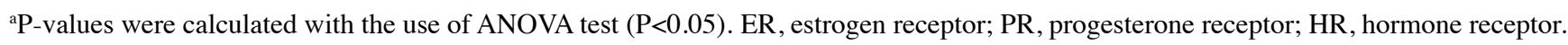

methylated in early primary breast tumors. These genes were composed of previously reported and novel genes in breast cancer. From the methylated target genes of Tommasi and colleagues (10), we found that 60/81 genes matched our data in breast cancer. This observation suggested that our strategy for selection of putative methylation biomarkers was sound. For clinical validation, we narrowed down the number of genes to three, $L H X 2, W T 1$ and $O T P$, through step-wise selection procedure (described in the results section in this study) which demonstrated a higher level and frequency of methylation in these 3 genes in breast tumor tissue when compared to adjacent normal tissue.

Notably our finding of $L H X 2$ and WT1, are also present in the previous gene list (10), indicating that an affinity-based CpG methylation microarray with methylated DNA binding domain of MBD2t or MBD2/MBD3L1 complex is a powerful approach for global methylation mapping.
Considering that one of the ultimate goals of tumor specific molecular markers is to detect tumors non-invasively in an early stage of development, tumor-specific DNA methylation might be the appropriate candidate for this purpose since aberrant methylation at $\mathrm{CpG}$ islands occurs frequently and is an early epigenetic event in tumorigenesis $(5,15,16)$. Conceptually a promising methylation marker with the purpose of early cancer detection is thought to be retaining the tendency of methylation positivity across all tumor types, and from early to advanced states regardless of clincopathological status including stage. Some portion of cell-free DNA in serum or plasma is thought to have originated from tumor cells undergoing either apoptosis or necrosis in cancer patients (17-19). Accordingly, identification of tumor-specifically methylated sites of genes is a critical step in the sequential process of molecular marker development for early cancer detection in body fluids including blood derivatives from breast cancer 


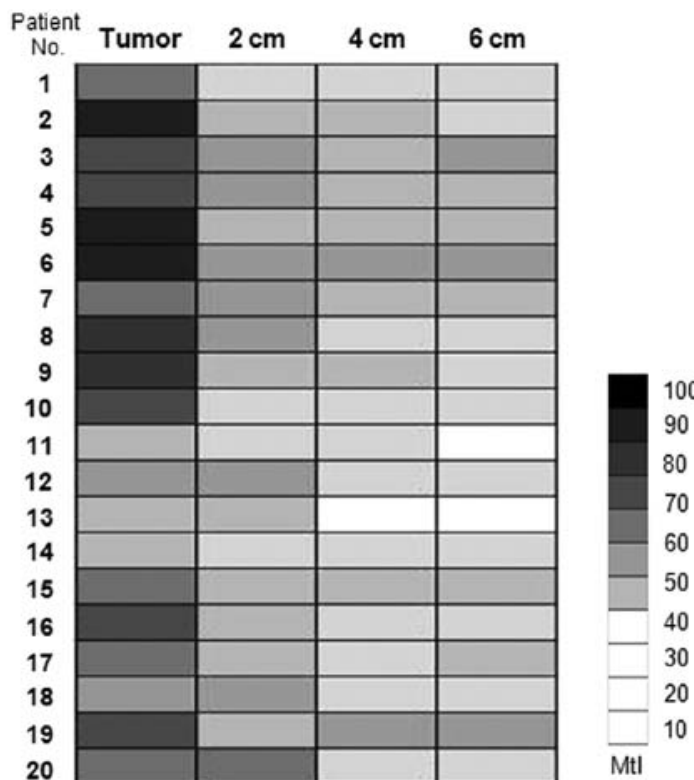

Figure 4. Methylation status of OTP gene in breast tumors and adjacent histologically normal tissues. Breast tissue was obtained from tumor and its surrounding zones 2, 4 and $6 \mathrm{~cm}$ from the grossly visible tumor boundary from patients who underwent mastectomy. The MtI of each sample is depicted by gradient black scale (right). Each row and column represents individual patients $(n=20)$ and MtIs from tumor $(\mathrm{T})$ and adjacent normal tissue $(2,4$ and $6 \mathrm{~cm})$, respectively.

patients. Therefore, we postulate that the OTP gene would be a strong candidate marker for early detection considering its methylation frequency (sensitivity/specificity) and the early time of occurrence.

First, this gene showed methylation in $100 \%$ of our 39 primary tumor samples. Second, although the level of methylation is lower than that of matched-primary tumor tissue, there is a high frequency of methylation positivity that clearly appears in $87 \%$ of tumor-adjacent normal tissue when given the cut-off values are used (Fig. 3 and Table IV).

To further assess the extent of aberrant methylation of the $O T P$ gene in adjacent normal tissues, we examined the methylation status of the OTP gene quantitatively in 20 cases of available adjacent normal tissues at various distances away from the tumor lesion boarder. Aberrant methylation of the OTP gene was extended up to $6 \mathrm{~cm}$ away from the primary tumor (Fig. 4). In addition, we could not find a significant correlation between $O T P$ gene methylation status and various clinicopathological characteristics (Table V). This suggests that this gene may gain aberrant methylation at a very early stage and be an indicator of predisposition in precancerous regions due to a field methylation effect.

Most gene methylation is barely detectable in the serum or plasma of healthy individuals. Recently, several groups, using a panel of multiple genes which are well known to be tumorassociated, have demonstrated the prevalence of methylation positive genes in sera or plasma from breast cancer patients (20-27). Nevertheless, current multiple gene panel assays still lack the necessary sensitivity for application in the clinical setting.

To our knowledge, the OTP gene has never been reported to be linked to DNA methylation modification in breast cancer.
Taken together, our findings strengthen the possibility of $O T P$ gene methylation as a biomarker candidate for early detection of breast cancer. The OTP gene has been described to be important for brain development $(28,29)$. In addition, Wu and colleagues have recently reported that $O T P$ is frequently methylated in astrocytomas (30). However, little is known about the biological function of this novel gene in cancer.

In summary, we demonstrated that microarray analysis using a minimum size of methylated DNA binding domain for methylated DNA enrichment successfully works for genomic level methylation profiling. With this approach we have identified the $O T P$ gene which can provide an additive sensitivity to current set of candidate genes for early detection of breast cancer.

More sample validation and defining a specific region or regions of $O T P$ that are methylated will be necessary to determine if the methylation status of this gene can be utilized as a biomarker for large scale, early detection of breast cancer.

\section{Acknowledgements}

This study was supported by a grant from the National R\&D Program for Cancer Control, Ministry of Health \& Welfare, Republic of Korea (no. 0720560).

\section{References}

1. Althuis MD, Dozier JM, Anderson WF, Devesa SS and Brinton LA: Global trends in breast cancer incidence and mortality 1973-1997. Int J Epidemiol 34: 405-412, 2005.

2. Coughlin SS and Ekwueme DU: Breast cancer as a global health concern. Cancer Epidemiol 33: 315-318, 2009.

3. Esteller M: Cancer epigenetics: DNA methylation and chromatin alterations in human cancer. Adv Exp Med Biol 532: 39-49, 2003.

4. Watanabe Y and Maekawa M: Methylation of DNA in cancer. Adv Clin Chem 52: 145-67, 2010.

5. Laird PW: The power and the promise of DNA methylation markers. Nat Rev Cancer 3: 253-266, 2003.

6. Fackler MJ, McVeigh M, Evron E, Garrett E, Mehrotra J, Polyak K, Sukumar S and Argani P: DNA methylation of RASSF1A, HIN-1, RAR-beta, Cyclin D2 and Twist in in situ and invasive lobular breast carcinoma. Int J Cancer 107: 970-975, 2003.

7. Fujikane T, Nishikawa N, Toyota M, Suzuki H, Nojima M, Maruyama R, Ashida M, Ohe-Toyota M, Kai M, Nishidate T, Sasaki Y, Ohmura T, Hirata K and Tokino: Genomic screening for genes upregulated by demethylation revealed novel targets of epigenetic silencing in breast cancer. Breast Cancer Res Treat 122: 699-710, 2010.

8. Hill VK, Ricketts C, Bieche I, Vacher S, Gentle D, Lewis C, Maher ER and Latif F: Genome-wide DNA methylation profiling of $\mathrm{CpG}$ islands in breast cancer identifies novel genes associated with tumorigenicity. Cancer Res 71: 2988-2999, 2011.

9. Nass SJ, Herman JG, Gabrielson E, Iversen PW, Parl FF, Davidson NE and Graff JR: Aberrant methylation of the estrogen receptor and E-cadherin 5'-CpG islands increases with malignant progression in human breast cancer. Cancer Res 60: 4346-4348, 2000.

10. Tommasi S, Karm DL, Wu X, Yen Y and Pfeifer GP: Methylation of homeobox genes is a frequent and early epigenetic event in breast cancer. Breast Cancer Res 11: R14, 2009.

11. Veeck J, Noetzel E, Bektas N, Jost E, Hartmann A, Knüchel R and Dahl E: Promoter hypermethylation of the SFRP2 gene is a high-frequent alteration and tumor-specific epigenetic marker in human breast cancer. Mol Cancer 7: 83, 2008.

12. Moon YH, Oh TJ, Kim NY, Kim MS and An SW: Methylated DNA isolation. Am Biotechnol Lab 27: 23-25, 2009.

13. Dworkin AM, Huang TH and Toland AE: Epigenetic alterations in the breast: Implications for breast cancer detection, prognosis and treatment. Semin Cancer Biol 19: 165-171, 2009. 
14. Widschwendter M and Jones PA: DNA methylation and breast carcinogenesis. Oncogene 21: 5462-5482, 2002.

15. Krassenstein R, Sauter E, Dulaimi E, Battagli C, Ehya H, Klein-Szanto A and Cairns P: Detection of breast cancer in nipple aspirate fluid by $\mathrm{CpG}$ island hypermethylation. Clin Cancer Res 10: 28-32, 2004

16. Patel A, Groopman JD and Umar A: DNA methylation as a cancer-specific biomarker: from molecules to populations. Ann N Y Acad Sci 983: 286-297, 2003.

17. Fleischhacker $M$ and Schmidt B: Circulating nucleic acids (CNAs) and cancer: a survey. Biochim Biophys Acta 1775: 181-232, 2007.

18. Schwarzenbach H, Stoehlmacher J, Pantel K and Goekkurt E: Detection and monitoring of cell-free DNA in blood of patients with colorectal cancer. Ann N Y Acad Sci 1137: 190-96, 2008.

19. Stroun M, Lyautey J, Lederrey C, Olson-Sand A and Anker P: About the possible origin and mechanism of circulating DNA apoptosis and active DNA release. Clin Chim Acta 313: 139-142, 2001.

20. Dulaimi E, Hillinck J, Ibanez de Caceres I, Al-Saleem T and Cairns P: Tumor suppressor gene promoter hypermethylation in serum of breast cancer patients. Clin Cancer Res 10: 6189-6193, 2004.

21. Hu XC, Wong IH and Chow LW: Tumor-derived aberrant methylation in plasma of invasive ductal breast cancer patients: clinical implications. Oncol Rep 10: 1811-1815, 2003.

22. Jing F, Zhang J, Tao J, Zhou Y, Jun L, Tang X, Wang Y and Hai H: Hypermethylation of tumor suppressor genes BRCA1, p16 and 14-3-3sigma in serum of sporadic breast cancer patients. Onkologie 30: 14-19, 2007.

23. Mirza S, Sharma G, Prasad CP, Parshad R, Srivastava A, Gupta SD and Ralhan R: Promoter hypermethylation of TMS1, BRCA1, ERalpha and PRB in serum and tumor DNA of invasive ductal breast carcinoma patients. Life Sci 81: 280-287, 2007.
24. Sharma G, Mirza S, Prasad CP, Srivastava A, Gupta SD and Ralhan R: Promoter hypermethylation of p16INK4A, p14ARF, CyclinD2 and Slit2 in serum and tumor DNA from breast cancer patients. Life Sci 80: 1873-1881, 2007.

25. Shukla S, Mirza S, Sharma G, Parshad R, Gupta SD and Ralhan R: Detection of RASSF1A and RARbeta hypermethylation in serum DNA from breast cancer patients. Epigenetics 1: 88-93, 2006.

26. Taback B, Giuliano AE, Lai R, Hansen N, Singer FR, Pantel K and Hoon DS: Epigenetic analysis of body fluids and tumor tissues: application of a comprehensive molecular assessment for early-stage breast cancer patients. Ann N Y Acad Sci 1075: 211-221, 2006

27. Tan SH, Ida H, Lau QC, Goh BC, Chieng WS, Loh M and Ito Y: Detection of promoter hypermethylation in serum samples of cancer patients by methylation-specific polymerase chain reaction for tumour suppressor genes including RUNX3. Oncol Rep 18: 1225-1230, 2007

28. Acampora D, Postiglione MP, Avantaggiato V, Di Bonito M and Simeone A: The role of Otx and Otp genes in brain development. Int J Dev Biol 44: 669-677, 2000.

29. Ryu S, Mahler J, Acampora D, Holzschuh J, Erhardt S, Omodei D, Simeone A and Driever W: Orthopedia homeodomain protein is essential for diencephalic dopaminergic neuron development. Curr Biol 17: 873-880, 2007.

30. Wu X, Rauch TA, Zhong X, Bennett WP, Latif F, Krex D and Pfeifer GP: $\mathrm{CpG}$ island hypermethylation in human astrocytomas. Cancer Res 70: 2718-2727, 2010. 